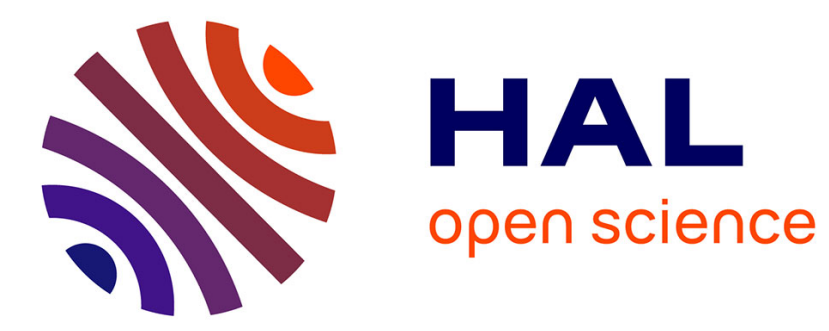

\title{
Autocorrélation interférométrique monocoup d'impulsions femtosecondes
}

Franck Salin, Patrick Georges, Gilles Le Saux, Gérard Roger, Alain Brun

\section{To cite this version:}

Franck Salin, Patrick Georges, Gilles Le Saux, Gérard Roger, Alain Brun. Autocorrélation interférométrique monocoup d'impulsions femtosecondes. Revue de Physique Appliquée, 1987, 22 (12), pp.1613-1618. 10.1051/rphysap:0198700220120161300 . jpa-00245720

\section{HAL Id: jpa-00245720 https://hal.science/jpa-00245720}

Submitted on 1 Jan 1987

HAL is a multi-disciplinary open access archive for the deposit and dissemination of scientific research documents, whether they are published or not. The documents may come from teaching and research institutions in France or abroad, or from public or private research centers.
L'archive ouverte pluridisciplinaire HAL, est destinée au dépôt et à la diffusion de documents scientifiques de niveau recherche, publiés ou non, émanant des établissements d'enseignement et de recherche français ou étrangers, des laboratoires publics ou privés. 


\title{
Autocorrélation interférométrique monocoup d'impulsions femtosecondes
}

\author{
F. Salin, P. Georges, G. Le Saux, G. Roger et A. Brun \\ Institut d'Optique Théorique et Appliquée, Centre Universitaire d'Orsay, Bâtiment 503, B.P. 43, 91406 Orsay \\ Cedex, France
}

(Reçu le 12 juin, révisé le 4 septembre, accepté le 18 septembre 1987)

\begin{abstract}
Résumé. - Nous présentons dans cet article une nouvelle méthode de caractérisation d'impulsions laser femtosecondes. Celle-ci est basée sur l'analyse du profil spatial du faisceau produit par doublage de fréquence lorsque deux impulsions se croisent dans un cristal non linéaire. Si ce profil est enregistré avec une précision interférométrique, la fonction d'autocorrélation est modulée par un système de franges. Cette fonction d'autocorrélation interférométrique monocoup permet d'obtenir la durée d'une impulsion prise isolément.
\end{abstract}

\begin{abstract}
A new method for the characterization of femtosecond laser pulses is reported in this article. We analyse the spatial shape of the second harmonic beam produced when two pulses cross in a non linear crystal. With an interferometric resolution, the autocorrelation function is modulated by interference fringes. This interferometric single-shot autocorrelation allows us to obtain the duration of a single pulse.
\end{abstract}

\section{Introduction.}

La production d'impulsions femtosecondes est maintenant chose courante dans de nombreux laboratoires de par le monde. On sait obtenir des impulsions de moins de $30 \mathrm{fs}$ [1] directement à la sortie d'une cavité laser et de $6 \mathrm{fs}$ après compression [2]. De même, le domaine spectral des impulsions femtosecondes s'étend de $200 \mathrm{~nm}$ [3] à 10,6 $\mu \mathrm{m}$ [4]. Pourtant très 'peu de progrès ont été réalisés pour améliorer les méthodes et les systèmes de caractérisation de ces impulsions. La plupart des laboratoires se contentent de la méthode classique de l'autocorrélation d 'ordre deux en intensité obtenue en faisant se croiser deux impulsions dans un cristal non linéaire et en mesurant la quantité de signal produit à l'harmonique deux en fonction du retard entre les deux impulsions. Quelques auteurs ont proposé d'autres méthodes. En particulier, Diels et al. étudient depuis plusieurs années les possibilités offertes par les fonctions d'autocorrélations enregistrées avec une précision interférométrique. Ils ont montré très récemment $[5,6]$ qu'en utilisant une méthode numérique on peut, à partir de l'enregistrement du spectre des impulsions, de la fonction d'autocorrélation en intensité et de la fonction d'autocorrélation interférométrique, remonter à la forme de l'impul- sion et obtenir des indications sur la répartition de la phase. Cette méthode est très attrayante mais reste relativement complexe et surtout nécessite des mesures (autocorrélations) qui ne peuvent être obtenues qu'à l'aide d'un très grand nombre (plusieurs millions) d'impulsions successives. Elle ne peut donc s'appliquer qu'aux lasers dont la stabilité peut être considérée comme très bonne. Dans le cas des lasers à faible taux de répétition la reproductibilité des impulsions est mauvaise. Il faut donc se tourner vers les méthodes dites « monocoup ».

En 1977, Jansky et al. [7] ont développé une technique permettant d'obtenir la fonction d'autocorrélation d'ordre 2 à l'aide d'une seule impulsion de quelques picosecondes. Cette méthode est basée sur l'analyse du profil spatial du faisceau à la fréquence double obtenue quand deux faisceaux se croisent dans un cristal non linéaire [8]. Récemment, nous avons étendu cette technique à des impulsions de moins de 60 fs [9]. Dans cet article, nous allons montrer théoriquement la possibilité de combiner les avantages des autocorrélations interférométriques et des techniques « monocoup » pour caractériser des impulsions uniques de moins de 100 fs. Nous présenterons ensuite les premiers résultats expérimentaux et discuterons des limitations introduites par le détecteur utilisé. 


\section{Approche théorique.}

2.1 Autocorrélateur Classique. - Pour mesurer la durée des impulsions émises par un laser femtoseconde, on enregistre généralement leur fonction d'autocorrélation d'ordre 2 en intensité. Pour cela, on mesure la quantité de lumière produite par doublage de fréquence dans un cristal non linéaire (KDP par exemple) en fonction du retard $\tau$ entre les deux impulsions incidentes sur le cristal. En notant :

$$
E_{j}(t)=A_{j}(t) \exp (i \omega t) \quad j=1,2
$$

les champs électriques des impulsions (supposées identiques), on peut montrer que l'intensité du signal du second harmonique est proportionnelle à :

$$
\begin{aligned}
F(\tau)=\int_{-\infty}^{+\infty} \mid[A(t) & \exp (i \omega t)+A(t-\tau) \times \\
& \times \exp (i \omega(t-\tau))]\left.\right|^{4} \mathrm{~d} t .
\end{aligned}
$$

Si les termes variant rapidement sont moyennés, on obtient la fonction d'autocorrélation d'ordre 2 « classique » :

$$
G_{2}(\tau)=\int_{-\infty}^{+\infty} I(t) I(t-\tau) \mathrm{d} t
$$

avec :

$$
I(t)=E(t) E^{*}(t) .
$$

Diels et al. ont montré [5] qu'en mesurant $F(\tau)$ avec une précision interférométrique (c'est-àdire, sans moyenner les termes variant en $\omega \tau$ oscillant rapidement), on obtient la fonction d'autocorrélation interférométrique de l'impulsion. Cette dernière est plus sensible à la forme et à la répartition de phase de l'impulsion que l'autocorrélation classique et permet d'avoir davantage d'informations sur le profil de l'impulsion.

2.2 Autocorrélateur monocoup. - Dans le cas des lasers à faible taux de répétition, on peut obtenir la fonction d'autocorrélation d'ordre 2 en intensité à l'aide d'une seule impulsion en enregistrant le profil spatial du faisceau à la fréquence double obtenu lorsque deux impulsions se croisent de façon synchrone dans un cristal de KDP [7-9]. Si les diamètres des deux faisceaux incidents sont suffisamment grands et que leurs profils spatiaux sont uniformes, on peut montrer que le profil spatial $S(x)$ du faisceau produit sur la bissectrice des faisceaux incidents (Fig. 1), est directement proportionnel à $G_{2}(2 \tau)$ :

$$
S(x) \propto \int_{-\infty}^{+\infty} I(t+\tau) I(t-\tau) \mathrm{d} t
$$

avec :

$$
x=\frac{c \tau}{2 n \sin (\phi / 2)}
$$

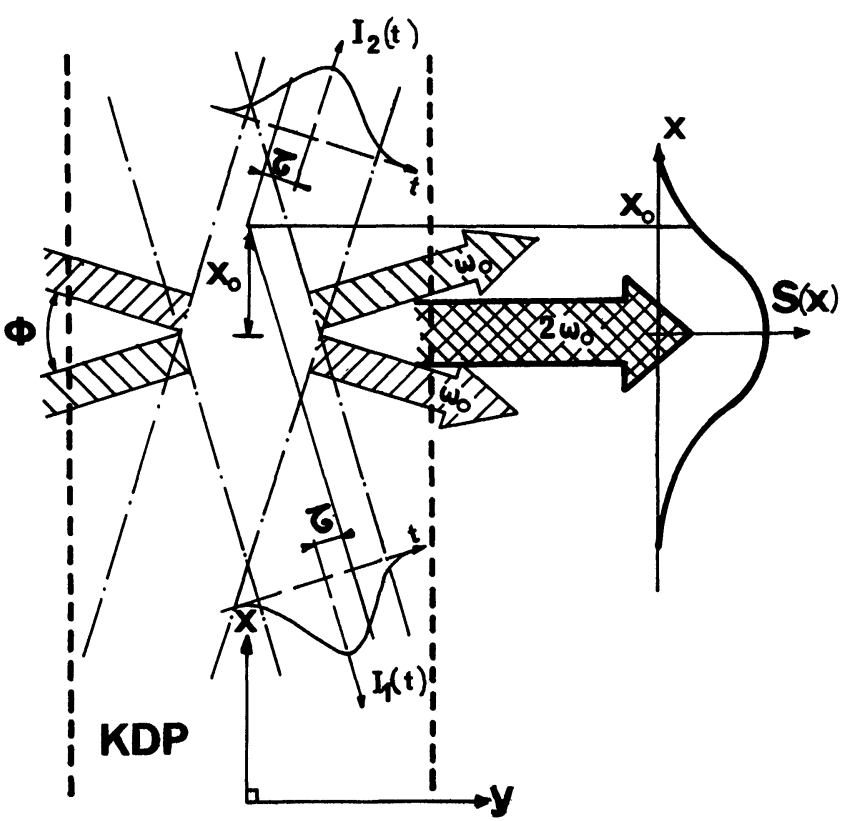

Fig. 1. - Schéma illustrant le croisement de deux faisceaux femtosecondes élargis dans un cristal de KDP.

[Sketch illustrating the interaction of two large femtosecond beams in a non-linear crystal.]

où $c$ est la vitesse de la lumière dans le vide, $n$ l'indice de réfraction du cristal, $\phi$ l'angle entre les deux faisceaux incidents et $2 \tau$ le retard entre les passages des maxima des deux impulsions à une abscisse $x$ dans le cristal de KDP. Récemment, nous avons enregistré par cette méthode la fonction d'autocorrélation en intensité d'une impulsion unique de $50 \mathrm{fs}$ à $\lambda=620 \mathrm{~nm}$ en faisant l'image du cristal de KDP sur une barette de photodiodes intensifiée [9].

Il faut noter que lorsque l'angle $\phi$ est suffisamment faible $\left(<1^{\circ}\right)$, l'efficacité du doublage est pratiquement identique dans les directions des deux faisceaux incidents et dans celle de la bissectrice. Il y a donc trois faisceaux proçuits à la fréquence double, mais le système optique utilisé dans notre expérience permettait de ne sélectionner que la lumière produite dans la direction de la bissectrice. Si le signal enregistré dans ce cas correspond bien à la fonction d'autocorrélation $G_{2}(2 \tau)$, il ne représente pas la répartition spatiale de l'intensité U.V. sur la face de sortie du cristal de KDP.

Nous allons maintenant tenir compte de ces trois faisceaux U.V. et exprimer la répartition spatiale de l'intensité lumineuse résultante.

2.3 AUTOCORRÉlATEUR MONOCOUP INTERFÉROMÉTRIQUE. - En notant $E_{1}(\omega), E_{2}(\omega)$, les champs électriques incidents sur le cristal, on peut calculer les champs $E_{3}(2 \omega), E_{4}(2 \omega)$ et $E_{5}(2 \omega)$ des trois faisceaux U.V. produits. 


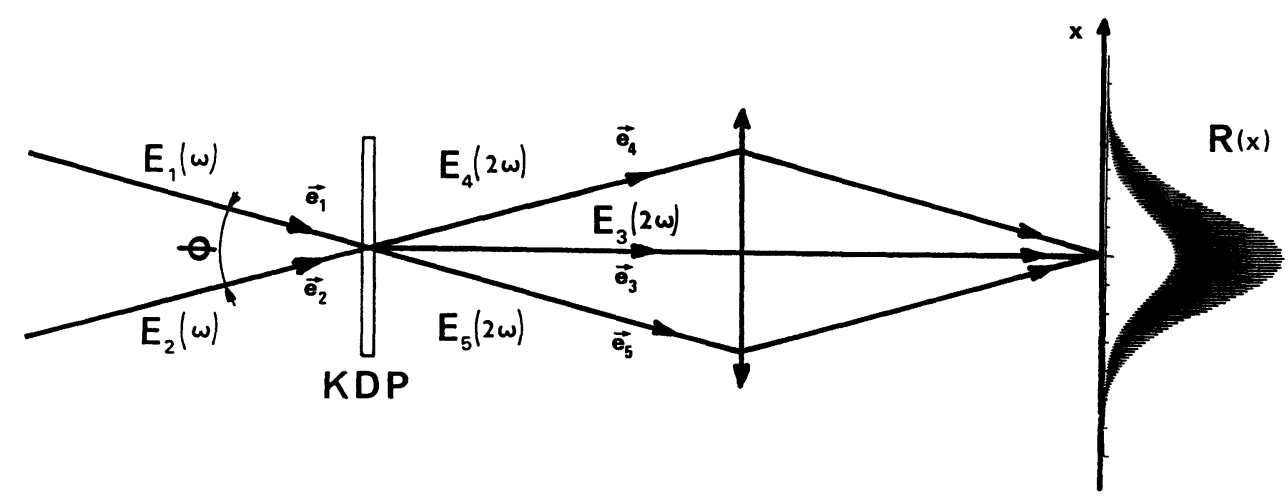

O.M.A.

Fig. 2. - Schéma précisant les directions des différents champs produits par doublage de fréquence lors du croisement de deux impulsions dans un cristal non linéaire.

[Scheme showing the directions of the beams produced by frequency doubling when two pulses cross in a non linear crystal.]

Dans le cas général du doublage de fréquence, le vecteur polarisation non linéaire $\mathbf{P}(2 \omega)$ s'écrit :

$$
\mathbf{P}(2 \omega)=\chi^{(2)}(-2 \omega, \omega, \omega) \mathbf{E}_{i}(\omega) \mathbf{E}_{j}(\omega)
$$

avec :

Dans le montage utilisé, les différentes combinaisons de $i$ et $j$ donnent lieu à trois champs à la fréquence $2 \omega$ qui rayonnent dans trois directions différentes (Fig. 2). En un point d'abscisse $x$ sur le cristal (correspondant à un retard $2 \tau$ entre les deux impulsions incidentes en ce point) ces champs s'écrivent :

$$
\begin{aligned}
\mathbf{E}_{k}(2 \omega, t, x)=A_{k}(2 \omega, t, x) \times \\
\quad \times \exp (i 2 \omega t) \mathbf{e}_{k} \quad k=3,4,5
\end{aligned}
$$

où $x$ est défini par (6).

En notant $\eta_{k}$ l'efficacité de doublage dans la direction $k$, les amplitudes $A_{k}$ s'écrivent :

$$
i=1,2 \text { et } j=1,2 \text {. }
$$

$$
\begin{aligned}
A_{4}(2 \omega, t, x)= & \eta_{4} \cdot A_{2}(\omega, t-\tau) \times \\
& \times A_{2}(\omega, t-\tau) \cdot \exp (i \omega 2 \tau) \\
A_{5}(2 \omega, t, x)= & \eta_{5} \cdot A_{1}(\omega, t+\tau) \times \\
\times & A_{1}(\omega, t+\tau) \cdot \exp (-i \omega 2 \tau) \\
A_{3}(2 \omega, t, x)= & \eta_{3}\left[A_{1}(\omega, t+\tau) \cdot A_{2}(\omega, t-\tau)+\right. \\
+ & \left.A_{2}(\omega, t-\tau) \cdot A_{1}(\omega, t+\tau)\right]
\end{aligned}
$$

On obtient les composantes $A_{x}$ et $A_{y}$ de l'amplitude de $A(2 \omega)$ du champ total à la fréquence $2 \omega$ en sommant les différents champs $\mathbf{E}_{k}$ produits. Soient :

$$
\begin{aligned}
& A_{x}(2 \omega, t, x)= \\
& =\eta_{4} I_{2}(t-\tau) \exp (i \omega 2 \tau) \cos (\phi / 2) \\
& +\eta_{5} I_{1}(t+\tau) \exp (-i \omega 2 \tau) \cos (\phi / 2) \\
& \quad+\eta_{3} \cdot 2 \cdot A_{1}(t+\tau) A_{2}(t-\tau)
\end{aligned}
$$

et :

$$
\begin{aligned}
& A_{y}(2 \omega, t, x)= \\
& =\eta_{4} I_{2}(t-\tau) \exp (i \omega 2 \tau) \sin (\phi / 2) \\
& \quad+\eta_{5} I_{1}(t+\tau) \exp (-i \omega 2 \tau) \sin (\phi / 2) .
\end{aligned}
$$

En considérant que l'angle $\phi$ est suffisamment petit pour que les efficacités de doublage $\eta_{k}$ soient égales, que $\cos (\phi / 2) \simeq 1$ et que $\sin (\phi / 2) \simeq 0$, l'intensité lumineuse à la fréquence $2 \omega$ s'écrit :

$$
\begin{aligned}
I(2 \omega, t, x)=A_{x} A_{x}^{*}= & \eta\left[I_{1}^{2}(t+\tau)+I_{2}^{2}(t-\tau)+4 I_{1}(t+\tau) I_{2}(t-\tau)+2 I_{1}(t+\tau) I_{2}(t-\tau) \times\right. \\
& \left.\times \cos (4 \omega \tau)+4 A_{1}(t+\tau) A_{2}(t-\tau)\left[I_{1}(t+\tau)+I_{2}(t-\tau)\right] \cos (2 \omega t)\right]
\end{aligned}
$$

$\mathrm{Si}$ on enregistre cette intensité sur un détecteur dont le temps de réponse est beaucoup plus grand que la durée de l'impulsion, on observe un signal $R(x)$ proportionnel à l'intégrale de $I(2 \omega, t, x)$ :

$$
R(x) \propto \int_{-\infty}^{+\infty} I(2 \omega, t, x) \mathrm{d} t
$$

soit :

$$
\begin{aligned}
R(x) \propto \xi_{1}+\xi_{2}+[4+2 \cos (4 \omega \tau)] & \int_{-\infty}^{+\infty} I_{1}(t+\tau) I_{2}(t-\tau) \mathrm{d} t+ \\
& +4 \cos (2 \omega t) \int_{-\infty}^{+\infty} A_{1}(t+\tau) A_{2}(t-\tau)\left[I_{1}(t+\tau)+I_{2}(t-\tau)\right] \mathrm{d} t
\end{aligned}
$$


avec :

$$
\xi_{j}=\int_{-\infty}^{+\infty} I_{j}^{2}(t) \mathrm{d} t
$$

En général, les deux faisceaux incidents sur le cristal ont la même intensité. $R(x)$ devient alors :

$$
\begin{aligned}
R(x) \propto 2 \xi+[4+2 \cos (4 \omega \tau)] & G_{2}(2 \tau)+ \\
& +4 \cos (2 \omega \tau) \int_{-\infty}^{+\infty} A(t-\tau) A(t+\tau)[I(t-\tau)+I(t+\tau)] \mathrm{d} t .
\end{aligned}
$$

On observe sur l'expression (18) une grande similitude entre les résultats obtenus pour les autocorrélateurs classiques (retard lentement variable dans le temps) et pour les autocorrélateurs monocoup. En effet, lorsque la résolution (temporelle pour les autocorrélateurs classiques ou spatiale pour les autocorrélateurs monocoup) du système de détection est suffisamment fine, on observe des franges d'interférences dont le contraste est modulé. Si par contre, le système ne permet pas de résoudre les franges, ces dernières sont moyennées et on retrouve dans les deux cas la fonction d'autocorrélation d'ordre 2 en intensité.

Nous avons simulé numériquement à partir de la formule (18), la fonction $R(x)$ pour une impulsion incidente dont l'amplitude du champ s'écrit :

$$
A(t)=\frac{1}{\operatorname{ch}\left(\frac{1,76 t}{T_{0}}\right)} .
$$

La figure 3 présente la fonction d'autocorrélation interférométrique monocoup d'une impulsion dont la largeur à mi-hauteur $T_{0}$ vaut $50 \mathrm{fs}$.

On observe une fonction modulée avec un inter-

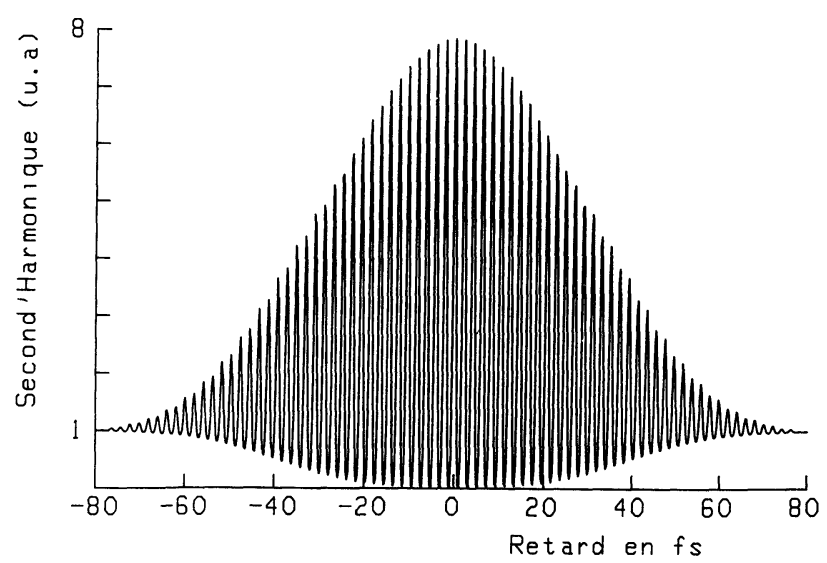

Fig. 3. - Fonction d'autocorrélation «interférométrique monocoup » théorique pour une impulsion en $1 / \mathrm{ch}^{2}$ d'une durée de $50 \mathrm{fs}$. Le retard porté en abscisse est $2 \tau$.

[Theoretical plot of a 50 fs pulse « single-shot interferometric» autocorrelation function $\left(1 / \mathrm{ch}^{2}\right.$ pulse shape). The temporal delay in abscissa is $2 \tau$.] frange correspondant à un retard de $\lambda / c$ où $\lambda$ est la longueur d'onde de l'impulsion incidente sur le cristal. A $620 \mathrm{~nm}$, l'interfrange correspond à 2,066 fs. On obtient donc un étalonnage très précis de la fonction mesurée. D'autre part, la largeur à mihauteur de l'enveloppe supérieure de la fonction $R(x)$ est égale à $1,32 T_{0}$.

\section{Observations expérimentales.}

Nous avons utilisé le montage décrit dans la référence [9] (Fig. 4). L'angle $\phi$ est diminué jusqu'à une valeur de l'ordre de 30 secondes d'arc. Le grandissement du système optique formant l'image du cristal de KDP sur le détecteur est d'environ 4. Dans le plan du détecteur, l'interfrange vaut donc environ $200 \mu \mathrm{m}$. L'ouverture du système optique permet de collecter les trois faisceaux U.V. produits au niveau du KDP. Il faut en effet, remarquer qu'aucun des trois faisceaux U.V. produits n'est spatialement modulé et que c'est seulement la recombinaison cohérente de ces trois faisceaux qui permet d'obtenir des franges sur le détecteur. Pour éviter que d'éventuels problèmes de cohérence spatiale ou d'inhomogénéité dans le profil du faisceau ne viennent perturber les mesures, nous avons mesuré la fonction d'autocorrélation interférométrique monocoup des impulsions directement produites par notre oscillateur femtoseconde [10]. Etant donné le très faible flux produit par doublage de fréquence dans le cristal de KDP, il faut utiliser un détecteur très sensible. Nous avons employé un analyseur optique multicanal (O.M.A. III) (barrette Réticon précédée d'un intensificateur de lumière à galette de microcanaux).

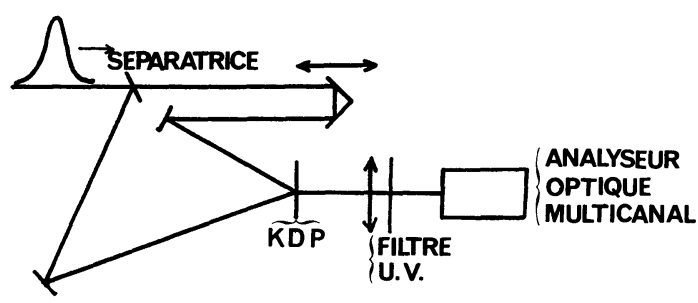

Fig. 4. - Montage expérimental.

[Experimental set-up.] 


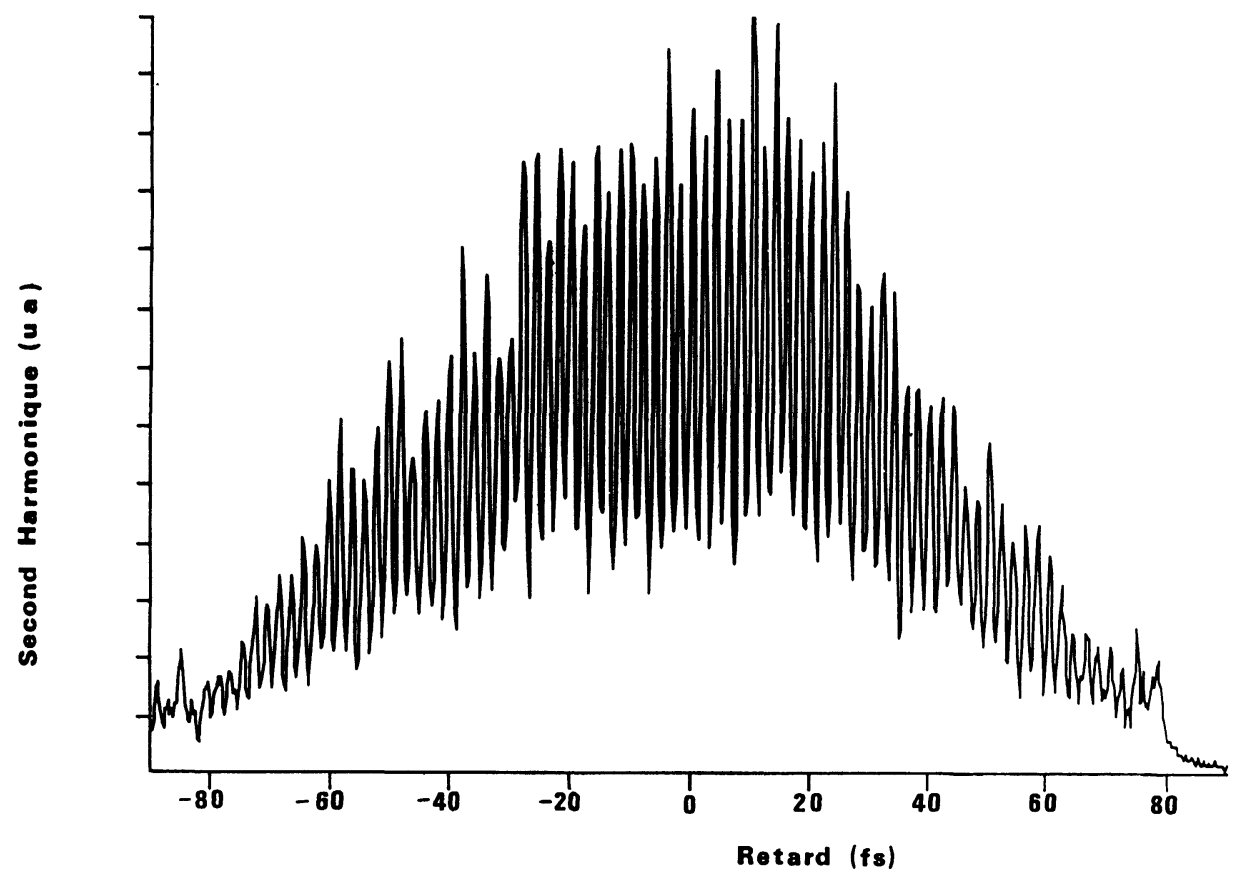

Fig. 5. - Enregistrement expérimental de la fonction d'autocorrélation interférométrique monocoup d'une impulsion de $70 \mathrm{fs}$ (signal intégré sur $10^{6}$ tirs).

[Experimental recording of a $70 \mathrm{fs}$ pulse single shot interferometric autocorrelation function (signal averaged over $10^{6}$ shots).]

La figure 5 présente un enregistrement expérimental obtenu à l'aide de l'autocorrélateur monocoup en moyennant le signal pendant $16 \mathrm{~ms}$ et en retranchant le fond continu. On peut remarquer que si l'enregistrement obtenu expérimentalement est bien modulé par des franges, sa forme générale est différente de celle prévue théoriquement (Fig. 3). En particulier, l'enveloppe inférieure des franges présente un maximum à $\tau=0$. Pour essayer d'expliquer ce désaccord, nous avons enregistré à l'aide de différents systèmes de détection la figure d'interférence produite par le croisement de deux faisceaux cohérents sur la surface des détecteurs. Dans tous les cas l'interfrange était très supérieur à la résolution spatiale des détecteurs. Une simple barrette Réticon nous a permis de vérifier que le contraste des franges était supérieur à $95 \%$. Par contre, ces mêmes franges visualisées à l'aide de l'OMA III présentent un contraste beaucoup plus faible et variant avec le pas des franges.

Pour un pas de franges équivalent à celui de la figure 3 , le contraste était inférieur à $30 \%$. La chute de contraste observée pourrait être due à la partie intensificateur de lumière du détecteur. En particulier, on peut penser qu'un certain nombre de photons transmis ou réfléchis par la photocathode peut créer des photoélectrons parasites qui engendrent un fond continu et diminuent le contraste. Nous avons essayé de prendre en compte cette diffusion parasite en supposant qu'une partie $a$ de l'inténsité lumineuse incidente crée un fond continu et que seule une partie $1-a$ participe au phénomène d'interférence.
Dans le cas de l'autocorrélateur monocoup interférométrique, on peut montrer que la fonction $R(x)$ devient :

$$
\begin{aligned}
R^{\prime}(x) & \propto 2 \xi+[4+2(1-a) \cos (4 \omega \tau)] \times \\
& \times G_{2}(2 \tau)+4(1-a) \cos 2 \omega \tau \int_{-\infty}^{+\infty} A(t-\tau) \\
& \times A(t+\tau)[I(t-\tau)+I(t+\tau)] \mathrm{d} t .
\end{aligned}
$$

La figure 6 présente l'évolution de la fonction $R^{\prime}(x)$ pour $a=0,7$. On peut remarquer que l'allure de cette fonction se rapproche de celle obtenue expérimentalement. On peut donc penser que des problèmes liés aux détecteurs nous empêchent d'observer la véritable fonction d'autocorrélation interférométrique.

Néanmoins nous avons vérifié que la largeur à mihauteur de l'enveloppe supérieure des franges mesurée par rapport au fond continu, est pratiquement indépendante du paramètre $a$. Nous pouvons donc utiliser l'enregistrement expérimental pour mesurer la durée d'une impulsion. Il suffit de compter le nombre de franges à mi-hauteur. La durée des impulsions estimée à partir de la figure 5 (en supposant un profil en intensité en $1 / \mathrm{ch}^{2}$ ) est d'environ $70 \mathrm{fs}$. Cette valeur est supérieure à celle que nous avons mesurée à l'aide d'un autocorrélateur classique : 50 fs. La différence observée peut provenir des phénomènes de dispersion de vitesse de groupe dans les éléments du montage (lame séparatrice, lentilles, etc...). 


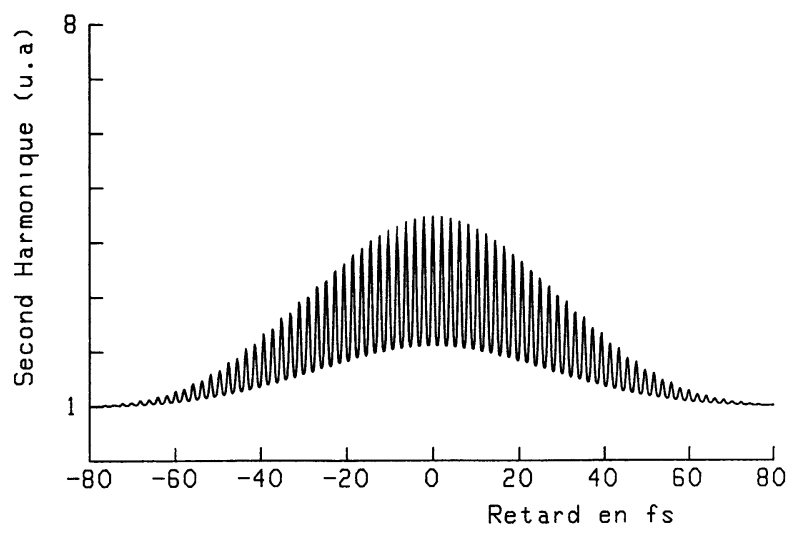

Fig. 6. - Fonction d'autocorrélation interférométrique monocoup théorique d'une impulsion de $50 \mathrm{fs}$ tenant compte d'une chute de contraste correspondant à $a=0,7$.

[Theoretical plot of a $50 \mathrm{fs}$ pulse single-shot interferometric autocorrelation function with a decrease of contrast $(a=0.7)$.]

\section{Conclusion.}

En résumé, nous avons calculé puis mesuré expérimentalement la répartition spatiale de l'intensité produite par doublage de fréquence dans un cristal non linéaire, lorsque deux impulsions se croisent en faisant un angle très faible. Nous avons montré que dans ce cas, cette répartition se présente sous la forme d'une fonction d'autocorrélation modulée par un système de franges. Cette fonction d'autocorrélation interférométrique monocoup possède beaucoup de similitudes avec les fonctions d'autocorrélations interférométriques classiques [5,6]. La méthode présentée ici permet d'obtenir ces informations sur une seule impulsion contrairement aux méthodes antérieures qui ne donnent qu'une valeur statistique sur un grand nombre d'impulsions.

Malheureusement, le faible flux lumineux disponible expérimentalement nous a obligé à utiliser un détecteur intensifié. Un phénomène parasite lié au détecteur nous a empêché d'obtenir des informations précises sur le profil exact des impulsions. Bien que la nature physique de ce phénomène soit encore très mal comprise, nous avons cependant pu mesurer avec précision la largeur temporelle des impulsions.

Ce travail a pu être réalisé grâce à un Contrat du Ministère de la Recherche et de la DRET (Division Optique).

\section{Bibliographie}

[1] Valdmanis, J. A., Fork, R. L. and Gordon, J. P., Opt. Lett. 10 (1985) 131.

[2] Brito Cruz, C. H., Fork, R. L., Shank, C. V., Conference on Lasers and Electro-optics (Baltimore) Avril 1987.

[3] Glownio, J. H., Argavalinghom, G., Sorokin, P. P. and Rothenburg, J. E., Opt. Lett. 11 (1986) 79.

[4] Rolland, C., Corkum, P. B., XV International Quantum Electronics Conference (Baltimore) Avril 1987.

[5] Diels, J. C., Fontaine, J. J., Mc Michael, I. C., Simoni, F., Appl. Opt. 24 (1985) 1270.
[6] Diels, J. C., Fontaine, J. J., Jamasbi, N., Ming LAI, MACKEY, J., Conference on Lasers and Electro-Optics (Baltimore) Avril 1987.

[7] Jansky, J., Corradi, G., Gyuzalian, R. N., Opt. Comm. 23 (1977) 293.

[8] Gyuzalian, R. N., Sogomonian, S. B., Horwath, Z. G., Opt. Comm. 29 (1979) 239.

[9] Salin, F., Georges, P., Roger, G. and Brun, A., Appl. Opt. 26, $\mathrm{n}^{\circ} 22$ (1987) sous presse.

[10] Salin, F., Grangier, P., Roger, G. and Brun, A., Phys. Rev. Lett. 56 (1986) 1132. 